\title{
A Patients with Hemidystonia and Blepharospasm Following Traumatic Injury
}

\author{
Yukihisa Suzuki ${ }^{1,2,3 *}$, Motohiro Kiyosawa1,4, Masato Wakakura ${ }^{5}$, Kiichi Ishiwata ${ }^{2}$, Keiichi Oda ${ }^{2}$ and Kenji Ishii
}

${ }^{1}$ Department of Ophthalmology and Visual Science, Tokyo Medical and Dental University, Tokyo, Japan

${ }^{2}$ Positron Medical Center, Tokyo Metropolitan Institute of Gerontology, Tokyo, Japan

${ }^{3}$ All Japan Federation of Social Insurance Associations Mishima Hospital, Mishima, Japan

${ }^{4}$ Kiyosawa Eye Clinic, Tokyo, Japan

5 Inouye Eye Hospital, Tokyo, Japan

\begin{abstract}
Dystonia is a syndrome characterized by sustained muscle contractions causing twisting or abnormal posturing. Secondary dystonia is well known subsequent to lesions of the brain. A patient was a 50 -year-old man, and he experienced traffic accident. He suffered bilateral hemiparesis by cervical vertebrae damage according to the accident. Although the organic brain damage was not found, left lateral hemidystonia and blepharospasm were observed after injury. We measured cerebral glucose metabolism of the patient using positron emission tomography with ${ }^{18} \mathrm{~F}$-fluorodeoxyglucose, and compared with 20 normal subjects (10 men and 10 women; mean age, $50.9 \pm$ 17.1 years). Cerebral glucose hypermetabolism was observed in both sides of the thalamus, putamen and primary somatosensory area of patient compared with normal subjects. The results of functional imaging studies of dystonia are often interpreted using the present anatomical model of information flow in the basal ganglia-thalamo-cortical motor circuit. Glucose hypermetabolism in the thalamus, putamen, and primary somatosensory area, may be reflecting existence of dystonic diseases.
\end{abstract}

Keywords: Blepharospasm; Brain metabolism; Dystonia; Hemidystonia; Positron emission tomography; Traumatic injury

Abbreviations: EB: Essential Blepharospasm; FDG: ${ }^{18}$ F-fluorodeoxyglucose; MRI: Magnetic Resonance Imaging; PET: Positron Emission Tomography; PSA: Primary Somatosensory Area; SPM: Statistical Parametric Mapping

\section{Introduction}

Dystonia is a syndrome characterized by sustained muscle contractions causing twisting or abnormal posturing [1]. Secondary dystonia is well known subsequent to lesions of the basal ganglia [2], thalamus, or brainstem [3]. Hemidystonia defined as involuntary, sustained posturing of the unilateral arm, leg, and face sometimes occurs following head trauma, with the cause of the contralateral basal ganglia or pontomesencephalic lesions [2]. On the other hand, blepharospasm is a form of focal dystonia characterized by involuntary movements of the musculature of the upper face. It has been reported that secondary blepharospasm is caused by infarctions in the thalamus [4], striatum [5], and brainstem [6], while there was no report of secondary blepharospasm which did not have structural lesions in these areas. We have been studying a rare case with hemidystonia and blepharospasm following traffic accident, which had no structural lesions in the brain. Here, we presented cerebral glucose metabolism characteristic of dystonia using positron emission tomography (PET) and ${ }^{18} \mathrm{~F}$-fluorodeoxyglucose (FDG).

\section{Materials and Methods}

\section{Case presentation}

A 50-year-old right-handed man without any past history of serious illness experienced traffic accident 10 years ago. Although the organic brain damage was not found, bilateral hemiparesis by cervical vertebrae damage was observed just after the accident. We observed tactile hypesthesia of left side of the body and loss of muscle strength of left. In addition, pain and hemidystonia of left arm and leg induced by tactile stimulus was also observed. He was conscious of the pain of the left arm induced by slight passive movements. Eye movements were full and smooth, but he required efforts to see left and upper left directions. Though no noticeable wounds were found on the head or face, he might have received the blow in the head or face. He came to feel dazzling after injury. Several months after the accident, he came to feel the difficulty of bilateral eyelids opening and twitching symptoms of the surrounding area of eyes. We diagnosed the condition of his eyes as blepharospasm, because we observed involuntary movements of the eyelids and abnormalities of blinking. Moreover, we surmised that his dazzling was photophobia, while blepharospasm patients frequently experience dazzling or eye pain [7].

The control subjects were 20 normal volunteers ( 10 men and 10 women; mean age, $50.9 \pm 17.1$ years). None of the participants had other neurologist-diagnosed neuro-psychiatric diseases or a family history of dystonic disorders. Neither the control subjects nor the patient had taken any neuro-psychiatric drugs.

Informed consent was obtained from each subject before study participation, and the Institutional Ethics Committee of Tokyo Metropolitan Institute of Gerontology approved the protocol. All the procedures conformed to the tenets of the Declaration of Helsinki.

\section{MRI scanning}

Magnetic resonance imaging (MRI) scans were obtained from a

*Corresponding author: Yukihisa Suzuki, Department of Ophthalmology and Visual Science, Tokyo Medical and Dental University, Graduated School, Tokyo, Japan, Tel: 81-3-3813-6111; Fax: 81-3-3818-7188; E-mail: suzuki@f8.dion.ne.jp

Received June 01, 2013; Accepted July 17, 2013; Published July 25, 2013

Citation: Suzuki Y, Kiyosawa M, Wakakura M, Ishiwata K, Oda K, et al. (2013) A Patients with Hemidystonia and Blepharospasm Following Traumatic Injury. J Neurol Neurophysiol 4: 153. doi:10.4172/2155-9562.1000153

Copyright: $\odot 2013$ Suzuki Y, et al. This is an open-access article distributed unde the terms of the Creative Commons Attribution License, which permits unrestricted use, distribution, and reproduction in any medium, provided the original author and source are credited. 
patient to screen for organic brain disorders with a 1.5-T Signa Horizon scanner (General Electric, Milwaukee, WI). Trans-axial images with T1-weighted contrast (3DSPGR; TR=9.2 ms, TE=2.0 ms, matrix size $=256 \times 256 \times 124$, voxel size $=0.94 \times 0.94 \times 1.3 \mathrm{~mm})$, T2-weighted contrast (First Spin Echo; TR=3000 ms, TE=100 ms, matrix size $=256$ $\times 256 \times 20$, voxel size $=0.7 \times 0.7 \times 6.5 \mathrm{~mm})$ FLAIR contrast $(2 \mathrm{D}$; $\mathrm{TR}=10000 \mathrm{~ms}, \mathrm{TE}=100 \mathrm{~ms}$, matrix size $=256 \times 256 \times 20$, voxel size $=0.7$ $\times 0.7 \times 6.5 \mathrm{~mm})$, and diffusion-weighted contrast $(2 \mathrm{D}$; TR $=6000 \mathrm{~ms}$, $\mathrm{TE}=$ minimum, matrix size $=128 \times 128 \times 20$, voxel size $=0.7 \times 0.7 \times 6.5$ $\mathrm{mm}$ ) were obtained.

\section{PET data acquisition}

PET scans were obtained using the SET 2400W scanner (Shimadzu, Kyoto, Japan) at the Positron Medical Center, Tokyo Metropolitan Institute of Gerontology. A bolus of $2.5 \mathrm{MBq} / \mathrm{kg}$ body weight FDG was injected intravenously into subjects. The subjects were then instructed to lie down with their eyes closed. A 6-min emission scan in the 3-dimensional acquisition mode was initiated $45 \mathrm{~min}$ after the injection, and 50 trans-axial images with an inter-slice interval of $3.125 \mathrm{~mm}$ were obtained (matrix size $=128 \times 128 \times 63$, voxel size $=2.0 \times 2.0 \times 3.125$ $\mathrm{mm}$ ). We monitored the subjects with a video camera during the scans and observed no extra movements in the face or other body parts. The tomographic images were reconstructed by the filtered backprojection method using a Butterworth filter (cut-off frequency, 1.25 cycles/ $\mathrm{cm}$; order, 2). Attenuation was corrected through performance of a transmission scan using a ${ }^{68} \mathrm{Ga} /{ }^{68} \mathrm{Ge}$ rotating source.

\section{Data processing and statistical analysis}

The PET images were processed using the statistical parametric mapping (SPM8) software [8], which was implemented in MATLAB (MathWorks, Sherborn, MA). Statistical parametric maps combine the general linear model and the theoretical Gaussian fields to make statistical inferences about regional effects. All PET images were spatially normalized to a standard template produced by Montreal Neurological Institute using a house-made template of FDG-PET images.

We defined 8.5-mm-diameter regions of interest on normalized PET images of both sides of the cerebellum, thalamus, caudate nucleus, putamen, primary somatosensory area (PSA), and we measured the cerebral glucose metabolism levels of each subject. Cerebral glucose metabolism level of each part was normalized to the mean of whole brain. We defined a significant change of cerebral glucose metabolism in a patient as an increase or decrease over the normal mean \pm 2 SDs [9].

\section{Results}

MRI revealed that there was no organic brain lesion in the patient. FDG-PET images of patient and normal subject are shown in Figure 1. Cerebral glucose hypermetabolism was observed in both sides of the thalamus, putamen and PSA of the patient compared with normal control subjects (Table 1).

\section{Discussion}

There have been reported hemidystonia or other dystonic diseases after head trauma. Krauss et al. reviewed patients with hemidystonia following head trauma, and CT or MRI revealed that all of the patients had contralateral basal ganglia or pontomesencephalic lesions [2]. Secondary blepharospasm is also caused by infarctions in the thalamus [4], striatum [5], and brainstem [6]. As far as we searched, there was no report of secondary hemidystonia or secondary blepharospasm, which did not have structural lesions in the brain. However, it has been reported that cervical dystonia following neck trauma [10] and oromandibular dystonia following facial injury [11] could occurred without brain lesions. Moreover, peripheral injuries to the face or neck have been implicated in many cases of focal or segmental dystonia $[12,13]$. The physiological mechanism of peripheral trauma-induced dystonia is not known, while some experimental studies suggest that peripheral injury can lead to reorganisation at cortical, subcortical and spinal cord levels resulting in motor dysfunction [11]. De Ceballos et al. observed that thermal injury to one limb in rats resulted in a reduction of leu-enkephalin concentration in the globus pallidum and striatum and met-enkephalin in the striatum [14]. From these observations, hemidystonia and blepharospasm of our patient might be caused by cervical vertebrae damage or facial bruise at the time of the traffic accident. There were several differences in clinical features between hemidystonia and blepharospasm of our patient. Hemidystonia was observed just after the accident, while the symptoms of blepharospasm gradually developed in several years after the accident. The blepharospasm was observed continuously without being affected by sensory stimuli. Hemidystonia of him was induced by tactile stimulus, but blepharospasm was not induced by the same stimulus. Therefore, it seems that blepharospasm was not associated with hemidystonia, and we suspected that blepharospasm is isolated symptom, not a part of hemidystonia.

We observed glucose hypermetabolism in both side of the thalamus, putamen and PSA of the patient. The results of functional imaging studies are often interpreted using the present anatomical model of information flow in the basal ganglia-thalamo-cortical motor

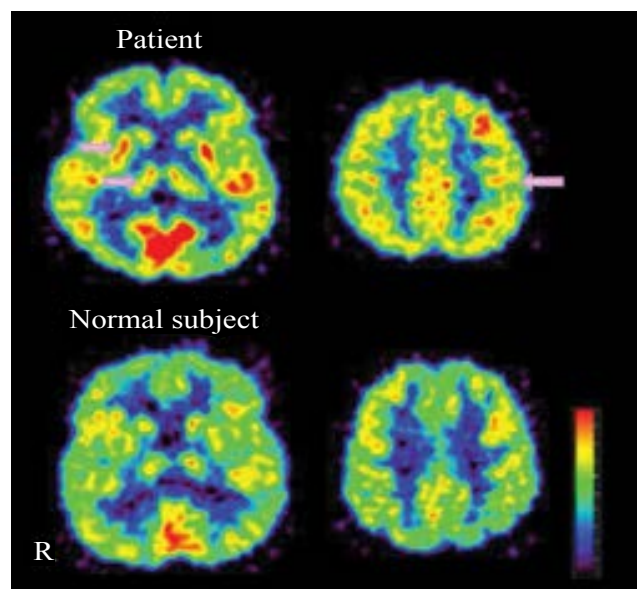

Figure 1: Images of positron emission tomography with 18F-fluorodeoxyglucose in a patient with hemidystonia and blepharospasm (up), and a normal subject (bottom). In the patient, glucose hypermetabolism in the thalamus, putamen, and primary somatosensory area are indicated by arrows.

\begin{tabular}{|c|c|c|c|c|c|c|}
\hline & & Thalamus & Caudate & Putamen & PSA & Cerebellum \\
\hline Patient & Right & $1.34^{*}$ & 1.27 & $1.51^{*}$ & $1.41^{*}$ & 1.10 \\
\hline & Left & $1.38^{*}$ & 1.26 & $1.54^{*}$ & $1.44^{*}$ & 1.08 \\
\hline $\begin{array}{c}\text { Average (SD) of } \\
\text { normal subjects }\end{array}$ & & & & & & \\
\hline & Right & 1.18 & 1.21 & 1.35 & 1.13 & 1.03 \\
\hline & Left & $\begin{array}{c}(0.075) \\
1.20\end{array}$ & $(0.094)$ & $(0.079)$ & $(0.070)$ & $(0.086)$ \\
\hline & & $(0.060)$ & $(0.089)$ & $(0.078)$ & $(0.056)$ & $(0.081)$ \\
\hline
\end{tabular}

Mean of whole brain $=1.0$

${ }^{*}$ Significant increase (over the normal average +2 SD)

PSA: primary somatosensory area

Table 1: Cerebral glucose metabolism in a patient. 
Citation: Suzuki Y, Kiyosawa M, Wakakura M, Ishiwata K, Oda K, et al. (2013) A Patients with Hemidystonia and Blepharospasm Following Traumatic Injury. J Neurol Neurophysiol 4: 153. doi:10.4172/2155-9562.1000153

Page 3 of 3

Basal ganglia-thalamo-cortical motor circuit of dystonia patients

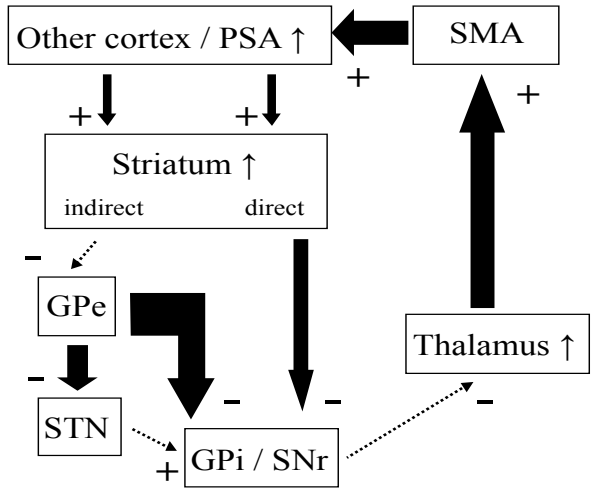

Figure 2: Hypothetical alterations of the basal ganglia-thalamo-cortical motor circuit. The striatum receives input from the primary somatosensory area (PSA) and from other motor and sensory cortex. The striatum projects by direct and indirect pathways to the major output structures of the basal ganglia, the globus pallidus interna (GPi) and substantia nigra reticulate $(\mathrm{SNr})$. An indirect pathway includes a striatal-globus pallidus externa (GPe) projection. Some GPe fibers project to the subthalamic nucleus (STN) and GPi/SNr, and other fibers project directly to the GPi/SNr. The GPi/SNr, which in turn, projects to the thalamus with a subsequent feedback to motor cortex, primarily the SMA. The effect of each structure on subsequent structures is to increase (+) or decrease (-) neuronal activity as indicated, adapted from Tempel et al. [15] and Garfen [22]. In dystonia patients, since glucose hypermetabolism in the basal ganglia, thalamus, and PSA was observed, it is surmised that the whole of the loop is being activated.

circuit $[15,16]$ (Figure 2). In our previous PET study, we also observed significant hypermetabolism in the thalamus, and we concluded that hyperactivity of the circuit may be relevant to the cause of essential blepharospasm [17]. Other studies also showed cerebral glucose hypermetabolism in the striatum and thalamus of patients with dystonia [18,19]. Moreover, there are several previous reports that activation in the somatosensory cortex was regarded during movement task in dystonic diseases $[20,21]$. It is surmised that cerebral glucose hypermetabolism in the thalamus, putamen, and PSA, was reflecting the existence of dystonic diseases in the present case. In the present case, we observed glucose hypermetabolism bilaterally, though he had a left lateral hemidystonia. The hemidystonia of him was observed only with tactile stimulus. Cerebral glucose metabolism was measured in the state that he was keeping quiet, and we did not observe the symptoms of hemidystonia during PET examinations. However, his symptom of bilateral blepharospasm was continued at the time of PET scanning. Bilateral glucose hypermetabolism in the thalamus, putamen and primary somatosensory area which we observed may reflect the symptom of blepharospasm.

\section{Conclusions}

We experienced a patient with hemidystonia and blepharospasm following traffic accident, and observed cerebral glucose hypermetabolism in the thalamus, putamen, and PSA.

\section{Acknowlegements}

This work was supported by the Benign Essential Blepharospasm Research Foundation (M. Kiyosawa) and Grants-in-Aid for Scientific Research No. 18791310 (YS) and No. 20591038 (K. Ishii) from the Japan Society for the Promotion of Science.

\section{References}

1. Wissel J, Poewe W (1992) Dystonia--a clinical, neuropathological and therapeutic review. J Neural Transm Suppl 38: 91-104.

2. Krauss JK, Mohadjer M, Braus DF, Wakhloo AK, Nobbe F, et al. (1992) Dystonia following head trauma: a report of nine patients and review of the literature. Mov Disord 7: 263-272.

3. Loher TJ, Krauss JK (2009) Dystonia associated with pontomesencephalic lesions. Mov Disord 24: 157-167.

4. Miranda M, Millar A (1998) Blepharospasm associated with bilateral infarcts confined to the thalamus: case report. Mov Disord 13: 616-617.

5. Grandas F, López-Manzanares L, Traba A (2004) Transient blepharospasm secondary to unilateral striatal infarction. Mov Disord 19: 1100-1102.

6. KostiÄ $\ddagger$ VS, StojanoviÄł-Svetel M, Kacar A (1996) Symptomatic dystonias associated with structural brain lesions: report of 16 cases. Can J Neurol Sci 23: 53-56.

7. Hallett M (2002) Blepharospasm: recent advances. Neurology 59: 1306-1312.

8. Friston KJ, Frith CD, Liddle PF, Frackowiak RS (1991) Comparing functional (PET) images: the assessment of significant change. J Cereb Blood Flow Metab 11: 690-699.

9. Suzuki Y, Nariai T, Kiyosawa M, Mochizuki M, Kimura Y, et al. (2012) Increased adenosine A1 receptor levels in hemianopia patients after cerebral injury: an application of PET using 11C-8-dicyclopropylmethyl-1-methyl-3propylxanthine. Clin Nucl Med 37: 1146-1151.

10. Samii A, Pal PK, Schulzer M, Mak E, Tsui JK (2000) Post-traumatic cervical dystonia: a distinct entity? Can J Neurol Sci 27: 55-59.

11. Sankhla C, Lai EC, Jankovic J (1998) Peripherally induced oromandibular dystonia. J Neurol Neurosurg Psychiatry 65: 722-728.

12. Koller WC, Wong GF, Lang A (1989) Posttraumatic movement disorders: a review. Mov Disord 4: 20-36.

13. Jankovic J (1994) Post-traumatic movement disorders: central and peripheral mechanisms. Neurology 44: 2006-2014.

14. de Ceballos ML, Baker M, Rose S, Jenner P, Marsden CD (1986) Do enkephalins in basal ganglia mediate a physiological motor rest mechanism? Mov Disord 1: 223-233.

15. Tempel LW, Perlmutter JS (1990) Abnormal vibration-induced cerebral blood flow responses in idiopathic dystonia. Brain $113: 691-707$.

16. Poston KL, Eidelberg D (2012) Functional brain networks and abnorma connectivity in the movement disorders. Neuroimage 62: 2261-2270.

17. Suzuki Y, Mizoguchi S, Kiyosawa M, Mochizuki M, Ishiwata K, et al. (2007) Glucose hypermetabolism in the thalamus of patients with essentia blepharospasm. J Neurol 254: 890-896.

18. Esmaeli-Gutstein B, Nahmias C, Thompson M, Kazdan M, Harvey J (1999) Positron emission tomography in patients with benign essential blepharospasm. Ophthal Plast Reconstr Surg 15: 23-27.

19. Galardi G, Perani D, Grassi F, Bressi S, Amadio S, et al. (1996) Basal ganglia and thalamo-cortical hypermetabolism in patients with spasmodic torticollis. Acta Neurol Scand 94: 172-176.

20. Preibisch C, Berg D, Hofmann E, Solymosi L, Naumann M (2001) Cerebra activation patterns in patients with writer's cramp: a functional magnetic resonance imaging study. J Neurol 248: 10-17.

21. Opavský R, Hluštík $P$, Otruba $P$, Kaňovský $P$ (2012) Somatosensory cortical activation in cervical dystonia and its modulation with botulinum toxin: an fMRI study. Int J Neurosci 122: 45-52.

22. Gerfen CR (1992) The neostriatal mosaic: multiple levels of compartmental organization. Trends Neurosci 15: 133-139. 